\title{
Neuropsychology of illiteracy
}

\author{
M. Rosselli \\ Unidad de Neurología, Hospital San Juan de Dios, Bogotá, Colombia \\ Correspondence to: M. Rosselli, Apartado Aéreo 17021, Bogotá, Colombia, South America
}

\begin{abstract}
It is proposed that analysis of illiteracy can not only discern the influence of schooling background on neuropsychological test performance, but also contributes to obtaining a better understanding about the cerebral organization of cognitive activity. Brain organization of cognition, and cognitive sequelae of brain pathology in illiterates are reviewed. It is concluded that: (1) cognitive abilities, as measured by standard neuropsychological tests, are significantly influenced by schooling background; and (2) educational and cultural variables may affect the degree (albeit, not the direction) of hemispheric dominance for language, and other cognitive abilities. A more bilateral representation of cognitive abilities in illiterates is hypothesized.
\end{abstract}

Keywords: Brain asymmetry - Cognition - Illiteracy - Neuropsychological assessment - Neuropsychology

\section{INTRODUCTION}

At least two different relevant aspects may be considered when studying the neuropsychology of illiteracy: (1) The analysis of illiteracy can help to discern the influence of schooling background on neuropsychological test performance. To distinguish the effects of brain damage from the influence of the subject's educational history represents a central problem in neuropsychological evaluation. Educational variables can significantly influence performance in neuropsychological test measures (e.g. Ardila et al., 1992a,b). (2) The study of illiteracy can contribute to obtaining a better and more accurate understanding of the cerebral organization of cognitive activity (Lecours et al., 1987a,b; Ardila et al., 1989b; Rosselli et al., 1990).

Illiterates represent a non-neglectable proportion of the world population (about one-third of people are illiterate; Unicef, 1985). Only a few centuries ago, reading and writing abilities were simply uncommon among the general population. Writing has only some 5000-6000 years in human history. It may be assumed that the acquisition of these skills may have somehow changed the brain organization of cognitive activity in general.

\section{BRAIN ORGANIZATION OF COGNITION IN ILLITERATES}

Several procedures have been applied to tackle the question about brain organization of cognition in illiterate persons.

Dichotic listening measures in illiterates

The role of the acquisition of reading and writing skills in the process of left brain specialization for language has

C1993 Rapid Communications of Oxford Ltd been the aim of several dichotic listening research studies (e.g. Damasio et al., 1979; Tzavaras et al., 1981; Castro and Morais, 1987).

Damasio et al. (1979) compared performance in three dichotic listening tasks in unschooled illiterates, semi-literates, and high school or college education individuals. In general, the two former groups ("dysliterates") scored at a significantly lower level than the high-educated subjects. When the stimuli consisted of pairs of digits or pairs of phonologically different meaningful words, a right ear advantage was found in all groups. When the stimuli were pairs of phonologically similar meaningful words, differing only in their initial consonants, a right ear advantage in the literate, and a left ear advantage in the dysliterate group was observed.

Tzavaras et al. (1981) studied the cerebral organization of right-handed Greek illiterates, and high-school control subjects, by testing their dichotic listening of pairs of digits. The two groups demonstrated a right ear advantage. The ear advantage index was significantly higher in the illiterate group when compared with the control group. The difference between the two ears was interpreted by the authors as a semi-extinction of the left ear, and as a nonability of language strategies of the right hemisphere. The authors concluded that it would be expected that education in general instigates several cognitive strategies for language which permit bi-hemispheric participation in linguistic function. If this is true, we can speculate that this harmonious bi-hemispheric control of language in educated subjects is not present among illiterates, who operate under the strong inhibitory influence of their left hemisphere. The results of this experiment, however, may have 
been influenced by the mean age differences between the experimental and control groups (Castro and Morais, 1987).

Castro and Morais (1987) tested right-handed females (illiterates, semi-literates, and literates) using a dichotic listening task. The three groups were homogeneous with regard to age. Subjects were presented with pairs of words phonologically similar and dissimilar, which differed either in the initial consonant only, or in at least the first three phonemes. The subjects' task was to report the word heard at the attended ear. A right ear advantage was observed in all groups for both similar and dissimilar pairs of words. There were no significant differences in the incidence of right ear advantage between literates and illiterates. Performance levels were higher for dissimilar than similar pairs and they increased from illiterates to semiliterates and from semi-literates to literates.

Results of these dichotic listening studies in illiterates are evidently confusing. It seems that illiterates and literates presented a right ear advantage with digits and dissimilar words. However, some illiterates may present a left ear advantage when similar words are used. At present, non-definitive conclusions can be obtained by dichotic listening studies. Methodological problems have been pointed out in these studies: the relationship between literacy and age, and lack of control for the orientation of attention (Castro and Morais, 1987). Evidently, more research is needed in this area in order to obtain sufficiently reliable conclusions.

\section{Neuropsychological assessment and illiteracy}

Educational attainment correlates to a high degree with scores on standard tests of intelligence. This correlation ranges from about 0.65 to 0.75 (Matarazzo, 1979), and in consequence, it could be considered that test performance correlations with IQ are in fact correlations with educational level. Correlations with verbal intelligence subtests are usually higher (from about 0.66 to 0.75 ) than correlations with performance intelligence subtests (from about 0.57 to 0.61 ). It could be argued that psychometric measures of intelligence are strongly biased by our current schooling system. In consequence, not only psychometric but also functional criteria of intelligence should always be taken into consideration (Pirozzolo, 1985).

Several studies have demonstrated a similarly strong association between educational level and performance on various neuropsychological measures (e.g. Finlayson et al., 1977; Ostrosky et al., 1985, 1986; Heaton et al., 1986; Bornstein and Suga, 1988; Ardila et al., 1992). However, some tests have been observed to be more sensitive to eductional variables (e.g. language tests) than others (e.g. the Wisconsin Card Sorting Test; Rosselli and Ardila, 1993). Extremely low scores in current neuropsychological tests are observed in illiterate people. Low scores in neuropsychological tests observed in illiterates may be partly due not only to differences in learning opportunities of those abilities that the examiner considers relevant (although, evidently, they are not the really relevant abilities for illiterates' survival), and to the fact that illiterates are not used to being tested (i.e. they have not learned how to behave in a testing situation), but also to the fact that testing itself represents a nonsense (non-relevant) situation.

Cornelious and Caspi (1987) found that educational level has a substantial relationship with performance in verbal meaning tests but was not systematically related to everyday problem solving (i.e. functional criterion of intelligence). Craik et al. (1987) observed that differences in memory loss during aging are related to socioeconomic status. Ardila and Rosselli (1989) reported that the educational variable was even more influential on neuropsychological performance than the age variable, and Albert and Heaton (1988) argued that, when education is controlled, there is no longer evidence of an age-related decline in verbal intelligence.

Ostrosky et al. $(1985,1986)$ applied a neuropsychological diagnostic test battery to a subject sample from two different socioeducational levels (high and low) in Mexico City. High socioeducational subjects performed better than low socioeducational subjects in all sections of the battery. However, differences were particularly notable in conceptual language tasks, and in programming of motor sequences, two abilities eventually associated with verbal learning history and writing skill training.

This significant schooling effect on neuropsychological test performance has been reported for different types of abilities. Some of them will be briefly mentioned.

Spatial abilities. Ardila et al. (1989b) administered a basic neuropsychological test battery to two extreme educational groups: illiterate and professional individuals. Subjects were matched according to sex and age. All the analyzed visuospatial tasks (copying a cube, a house, and Rey-Osterrieth Complex Figure; telling the time; recognizing superimposed figures; recognizing the national map; and drawing the plan of the room) showed highly significant differences between the two groups. In all these subtests, gender interacted with educational level. In the illiterate group men outperformed women, but no gender differences were observed in the professional sample.

Significant cross-cultural differences in perceptual abilities have been observed. Deregowski (1980) argues that in all societies there is a preference in children for drawing in two dimensions that persists if they do not have practice in three-dimensional drawings. Dawson (1967) suggested that general exposure to pictorial material might not be enough for learning pictorial representation, and some use of pictorial material is also required. Modiano et 
al. (1982) showed errors in Mexican Indian children in identifying color paintings and photographs of everyday objects. Hudson $(1960,1962)$ studied depth perception using pictures that contained figures of an elephant, an antelope and a man with a spear. It was observed that European children around 7-8 years old have a great deal of difficulty perceiving pictures as three-dimensional. However at around 12 years, virtually all can recognize three-dimensionality. African children (Bantu or Ghanian) did not perceive the three dimensions of the picture. Non-literate Bantu and Europeans responded to the picture as flat and not three-dimensional. On the other hand, hunting people with specific environmental demands usually present good visual discrimination and excellent spatial skills. For instance, the embedded figures test is better performed by cultural groups for whom hunting is important for survival (Berry, 1971, 1979).

Memory. Statistically significant differences between illiterates and professionals were also found in memory tests by Ardila et al. (1989b). For instance, professionals memorized a 10-word list after an average of 3.22 presentations; while illiterates required 6.55 repetitions. Significant differences were also found in the 10-word delay recall. There was a statistically significant interaction between age and educational level, with illiterates presenting a more notable variation across age groups. Immediate and delayed logical memory tests were also sensitive to educational level. Barltlett (1932) proposed that illiterates more frequently use procedures of rote learning while literate people refer to more active information integration procedures. Cole and Scribner (1974) observed that when memorizing information literates and illiterates make use of their own groupings to structure their recall; for instance high school subjects rely mainly on taxonomic categories, whereas illiterate bush farmers make little use of this principle. The authors argue that cultural differences in memorizing do not consist of the presence or absence of mnemonic techniques in general, but in the utilization of a specific technique: reorganization of to-be-remembered material. This particular strategy for recall could be tied to school learning experiences. Cole et al. $(1971 \mathrm{a}, \mathrm{b})$ compared Liberian Kpelle children with American children in the recall of spoken words and objects. They found a superior performance in the latter group. Kpelle subjects, on the other hand, showed a dissociation between verbal and non-verbal memory: they recalled objects better than spoken words. In Ardila et al.'s (1989b) study, differences between educational groups decreased sharply when no language mediation was used in the visual memory tasks.

Language. Language abilities have been strongly correlated with socioeducational level. Robinson (1974) observed that low socioeconomical parents use more non- verbal strategies in their relationship with their children. Bernstein (1974) points out that the language used by low socioeconomical people is less fluent and has a simpler grammatical structure; it relies much more on emotional than logical strategies. Bruner et al. (1966) suggested that rural unschooled children may lack symbolic representation skills because their linguistic ability is tied to the immediate context of the referent. They proposed that formal education facilitates the development of language into a fully symbolic tool. Lantz (1979), however, showed that rural unschooled children performed better than schooled Indian or American children in coding and decoding culturally relevant objects, such as grain, seeds, etc. Thus children without formal schooling are able to separate language symbols from the physical referent and to use those symbols for communicating accurately, but display of this ability depends upon the stimuli used (Laboratory of Comparative Human Cognition, 1983). Luria (1976) points out that the significance of schooling lies not just in the acquisition of new knowledge, but in the creation of new motives and formal modes of discursive verbal and logical thinking divorced from the immediate practical experience.

Praxic abilities. Education has been shown to be an important variable in motor performance subtests (Ostrosky et al., 1985). Rosselli et al. (1990) observed in illiterates differences according to educational level in the performance of buccofacial, ideomotor, and finger alternating movements subtests. Illiterate subjects presented some errors in these tasks while high educational level subjects did not present any mistakes. Interaction between education and age in performing buccofacial movements under verbal command was observed, and older illiterates presented the highest number of errors. In ideomotor praxis subtests a significant interaction between education and sex was observed. Illiterate women presented roughly double the mistakes of illiterate men. Fine finger movements were poorly performed by illiterate subjects in Rosselli et al.'s study. The absence of training and practice in fine movements (particularly writing) may account for the difference in fine movement test performance between the illiterates and high-educated subjects. Ostrosky et al. $(1985,1986)$ reported significant differences in performing programmed movements between subjects coming from different educational levels.

The literature reviewed in this section points to the importance of schooling effects on neuropsychological tests. Virtually all cognitive abilities measured by neuropsychological tests are sensitive to some degree to the subject's educational background. Without careful consideration of educational variables, neuropsychology runs the risk of finding brain pathology where there are only educational differences. Norms should be developed 
for low educational subjects. Neuropsychological testing should be given to illiterate subjects in order to find out their cognitive and behavioral profile and to design appropriate neuropsychological assessment procedures for this population.

\section{BRAIN DAMAGE AND ILLITERACY}

Two opposite points of view are found in the neuropsychological literature regarding the influence of education on brain organization of language. Cameron et al. (1971) reported that there is a lower frequency of aphasias associated with injuries of the left hemisphere among righthanded illiterate patients than among educated ones. The authors concluded that language is more bilaterally represented in the illiterate group. Damasio et al. (1976) claim that there is no qualitative or quantitative difference between the aphasias of educated and illiterate patients. The aphasia of literates or illiterates did not differ in expectancy rate, distribution of clinical types or semiological structure.

Matute's (1988) research studies provide some support to Damasio et al.'s conclusions. She compared three groups of right-handed Mexican subjects: brain-damaged illiterates, brain-damaged literates, and normal illiterates. An aphasia test was given to all three subject groups as part of a neuropsychological assessment. All left hemispheredamaged illiterate subjects presented aphasia, while no illiterates presented aphasia after right hemisphere damage. The aphasia was, however, less severe in the illiterate group than in the literate one. The literate group presented a higher number of errors, with lower scores in the aphasia subtests than the illiterate brain-damaged individuals.

Lecours et al. (1987a,b, 1988) studied some relationships between brain damage and schooling with regard to aphasic impairments of language. The authors concluded from their results that: (1) there was a greater right hemisphere language involvement in illiterates than in welleducated subjects; and (2) left stroke school-educated subjects seemed to be "sicker" than their illiterate counterparts, that is: (a) the classical symptoms of aphasia (suppression stereotype, jargonaphasia) are more apparent among left stroke literates than among left stroke illiterates; and (b) auditory comprehension was more frequently impaired among the left literate patients. Lecours et al. (1987b) studied also the influence of education on unilateral neglect syndrome. They analyzed a large sample of right-handed unilingual brain-damaged subjects: illiterates (left stroke and right stroke) and literates (left stroke and right stroke). Evidence of unilateral neglect syndrome was found in both left and right brain-damaged literates and illiterates. Their results provide no indication that tropisms were globally stronger depending on the side of the lesion or on the educational level of the subjects. Rosselli et al. (1985), however, reported a higher frequency of right hemi-spatial neglect in low-educated subjects.

Studies of brain-damaged illiterates when compared with brain-damaged literates lead to the following conclusions: (1) literacy does not change the dominance of the left hemisphere for language; illiterates as well as literates present aphasia most often after left brain damage, and not after right brain damage; (2) it seems, however, that the right hemisphere has more participation in language in illiterate subjects. There is a general consensus that leftdamaged literates present a higher number of errors in aphasia tests than left-damaged illiterates (Lecours et al., 1988; Matute, 1988), and that right-damaged illiterates present more frequently lower performance in aphasia tests than right brain-damaged literates (Lecours et al., 1987a,b).

\section{CONCLUSIONS}

It is evident that literacy is strongly reflected in the performance of those tasks used not only in psychological, but also neuropsychological evaluation. Very important cognitive consequences of learning to read and to write have been suggested: changes in visual perception, logical reasoning, and remembering strategies (Laboratory of Comparative Human Cognition, 1983). Even the influence of schooling on formal operational thinking has been pointed out (Laurendeau-Bendavid, 1977).

The analysis of performance of illiterate populations in neuropsychological measures suggests that cognitive abilities, as measured by standard neuropsychological tests, are significantly influenced by schooling. It is a mistake to assume that the inability to perform simple cognitive tasks, such as those incorporated in current neuropsychological test batteries, necessarily means abnormal brain function. The degree of literacy can often represent the crucial variable.

The influence of literacy seems to go further: literacy may somehow change the brain organization of cognition. We are far from completely understanding the influence of external variables on brain organization of cognitive activity. However it is a fact that educational and cultural variables may affect not only handedness (Ardila et al., 1989a; Bryden et al., 1993), but also the degree of hemispheric dominance for language (Matute, 1988; Lecours $e t$ al., 1988) and most likely other cognitive abilities. Studies on the consequences of brain damage in illiterate populations evidence a more bilateral representation not only for linguistic, but probably also for visuospatial abilities. Apparently, literacy does not change the direction of laterality in the brain organization of cognition, but the degree of this lateralization.

It can be assumed that the brain organization of cognition in prehistoric humans was closer to the organization observed in illiterate rather than schooled people. Writing 
has only a 5000-6000 year history, and obviously prehistoric humans were illiterate. Cultural knowledge and cognitive abilities mediated through written language are recent historical acquisitions. A more bilateral representation of not only linguistic but also visuospatial functions in prehistoric humans can be hypothesized.

\section{REFERENCES}

Albert MS and Heaton RK (1988) Intelligence testing. In: Geriatric Neuropsychology (Eds MS Albert and MB Moss), pp. 10-32. The Guilford Press, New York.

Ardila A and Rosselli M (1989) Neuropsychological characteristics of normal aging. Developmental Neuropsychology, 5, 307-320.

Ardila A, Ardila O, Bryden MP, et al. (1989a) Effects of cultural background and education on handedness. Neuropsychologia, 27, 893-898.

Ardila A, Rosselli M and Rosas P (1989b) Neuropsychological assessment in illiterates: Visuospatial and memory abilities. Brain and Cognition, 11, 147-166.

Ardila A, Rosselli M and Ostrosky F (1992a) Sociocultural factors in neuropsychological assessment. In: Handbook of Neuropsychological Assessment: A Biopsychosocial Perspective (Eds AE Puente and RJ McCaffrey), pp. 181-192. Academic Press, New York.

Ardila A, Rosselli M and Puente A (1992b) Neuropsychological Evaluation of the Spanish Speaker. Plenum, New York.

Barltlett FC (1932) Remembering. Cambridge University Press, London.

Berry JW (1971) Ecological and cultural factors in spatial perceptual development. Canadian Journal of Behavioral Sciences, 3, 324-336.

Berry JW (1979) Culture and cognition style. In: Perspectives in Cross-cultural Psychology (Eds A Marsella, RG Tharp, and TJ Ciborowski), pp.117-135. Academic Press, New York.

Bornstein RA and Suga LJ (1988) Educational level and neuropsychological performance in healthy elderly subjects. Developmental Neuropsychology, 4, 17-22.

Bruner J, Oliver R and Greenfield P (1966) Studies in Cognitive Growth. Wiley, New York.

Bryden MP, Ardila A and Ardila O (1993) Handedness in native Amazonians. Neuropsychologia, 31, 301-308.

Cameron RF, Currier RD and Haerer AF (1971) Aphasia and literacy. British Journal of Disorders of Communication, $\mathbf{6}$, 161-163.

Castro SL and Morais J (1987) Ear differences in illiterates. Neuropsychologia, 25, 409-417.

Cole M and Scribner S (1974) Culture and Thought. Wiley, New York.

Cole M, Frankel F and Sharp DW (1971a) The developmental of free recall learning in children. Developmental Psychology, 4, 109-123.

Cole M, Gay J, Glick J, et al. (1971b) The Cultural Context of Learning and Thinking. Basic Books, New York.

Cornelius SW and Caspi A (1987) Everyday problem solving in adulthood and old age. Psychology of Aging, 2, 144-153.

Craik FM, Byrd M and Swanson JM (1987) Patterns of memory loss in three elderly samples. Psychology of Aging, 2, 79-86.

Damasio AR, Castro-Caldas A, Grosso JT, et al. (1976) Brain specialization for language does not depend on literacy. Archives of Neurology, 33, 300-301.
Damasio H, Damasio AR, Castro-Caldas A, et al. (1979) Reversal for ear advantage for phonetically similar words in illiterates. Journal of Clinical Neuropsychology, 1, 331-338.

Dawson JLM (1967) Cultural and physiological influences upon spatial perceptual process in West Africa, Part 1. International Journal of Psychology, 2, 115-128.

Deregowski JB (1980) Illusions, Patterns and Pictures: A Cross Cultural Perspective. Academic Press, New York.

Finlayson NA, Johnson KA and Reitan RM (1977) Relation of level of education to neuropsychological measures in brain damaged and non-brain damaged adults. Journal of Consulting and Clinical Psychology, 45, 536-542.

Heaton RK, Grant I and Mathews C (1986) Differences in neuropsychological test performance associated with age, education and sex. In: Neuropsychological Assessment in Neuropsychiatric Disorders (Eds I Grant and KM Adams), pp. 108-120. Oxford University Press, New York.

Hudson W (1960) Pictorial depth perception in subcultural groups in Africa. Journal of Social Psychology, 52, 193-208.

Hudson W (1962) Cultural problems in pictorial perception. South African Journal of Sciences, 58, 189-195.

Laboratory of Comparative Human Cognition (1983) Culture and cognitive development. In: Handbook of Child Psychology. Vol. 1. History, Theories and Methods (Ed. P. Mussen), pp. 342-397. Wiley, New York.

Lantz D (1979) A cross-cultural comparison of communication abilities: Some effects of age, schooling and culture. International Journal of Psychology, 14, 171-183.

Laurendeau-Bendavid M (1977) Culture, schooling and cognitive development: A comparative study of children in French Canada and Rwanda. In: Piagetian Psychology: Cross Cultural Contributions (Ed. PR Dasen). Gardner, New York.

Lecours RL, Mehler J, Parente MA, et al. (1987a) Illiteracy and brain damage 1: aphasia testing in culturally contrasted populations (control subjects). Neuropsychologia, 25, 231-245.

Lecours RL, Mehler J, Parente MA, et al. (1987b) Illiteracy and brain damage 2: Manifestations of unilateral neglect in testing "auditory comprehension" with iconographic material. Brain and Cognition, 6, 243-265.

Lecours AR, Mehler J, Prente MA, et al. (1988) Illiteracy and brain damage III: A contribution to the study of speech and language disorders in illiterates with unilateral brain damage (initial testing). Neuropsychologia, 26, 575-589.

Luria AR (1976) Cognitive Development. Harvard University Press, Cambridge, MA.

Matarazzo JD (1979) Wechsler's Measurement and Appraisal of Adult Intelligence, 5th edn. Oxford University Press, New York.

Matute E (1988) El aprendizaje de la lectoescritura y la especi alizacion hemisferica para el lenguaje. In: Lenguaje oral y escrito (Eds A Ardila and F Ostrosky), pp. 310-338. Trillas, Mexico.

Modiano N, Maldonado LM and Villasana S (1982) Accurate perception of color illustrtions: Rates of comprehension in Mexican Indian children. Journal of Cross Cultural Psychology, 13, 490-495.

Ostrosky F, Canseco E, Quintanar L, et al. (1985) Sociocultural effects in neuropsychological assessment. International Journal of Neuroscience, 27, 53-66.

Ostrosky F, Quintanar L, Meneses S, et al. (1986) Actividad cognoscitiva y nivel sociocultural. Revista de Investigacion Clinica, 38, 37-42.

Pirozzolo FJ (1985) Mental retardation: An introduction. In: 
Handbook of Clinical Neurology. Vol 46. Neurobehavioral Disorders (Ed. JAM Frederiks), pp. 1-40. Elsevier, Amsterdam.

Robinson WP (1974) Social factors and language development in primary school children. In: Language Acquisition: Models and Methods (Eds R Huxley and E. Ingram). Academic Press, New York.

Rosselli M and Ardila A (1993) Effects of age, gender and socioeconomical level on the Wisconsin Card Sorting Test. The Clinical Neuropsychologist, 7, 145-154.

Rosselli M, Rosselli A, Vergara I and Ardila A (1985) The top- ography of the hemi-inattention syndrome. International Journal of Neuroscience, 20, 153-160.

Rosselli M, Ardila A and Rosas P (1990) Neuropsychological assessment in illiterates II: Language and praxic abilities. Brain and Cognition, 12, 281-296.

Tzavaras A, Kaprinis G and Gatzoyas A (1981) Literacy and hemispheric specialization for language: digit dichotic listening in illiterates. Neuropsychologia, 19, 565-570.

Witkin HA and Berry JW (1975) Psychological differentiation in cross-cultural perspective. Journal of Cross-Cultural Psychology, 6, 4-87. 


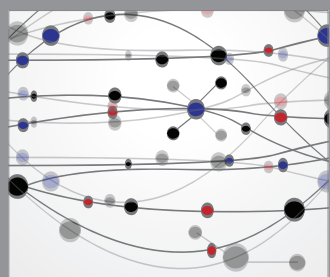

The Scientific World Journal
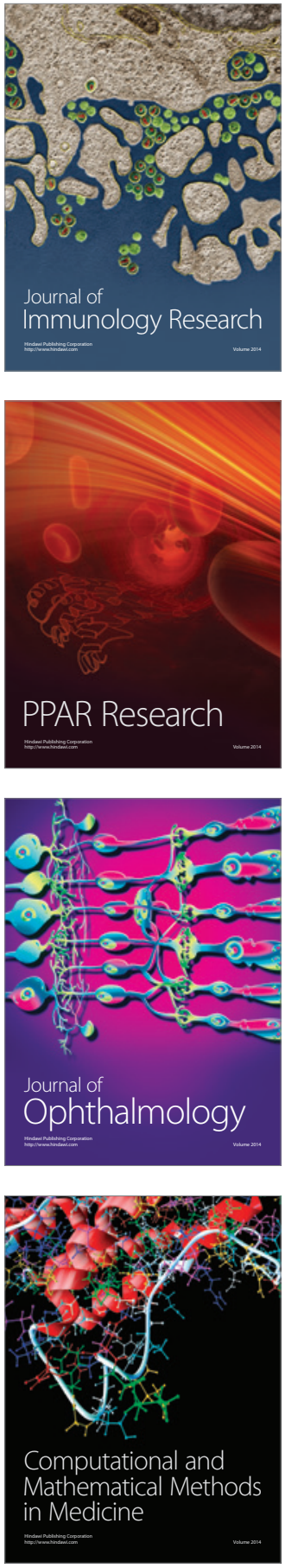

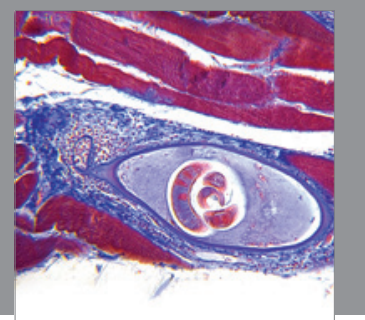

Gastroenterology

Research and Practice
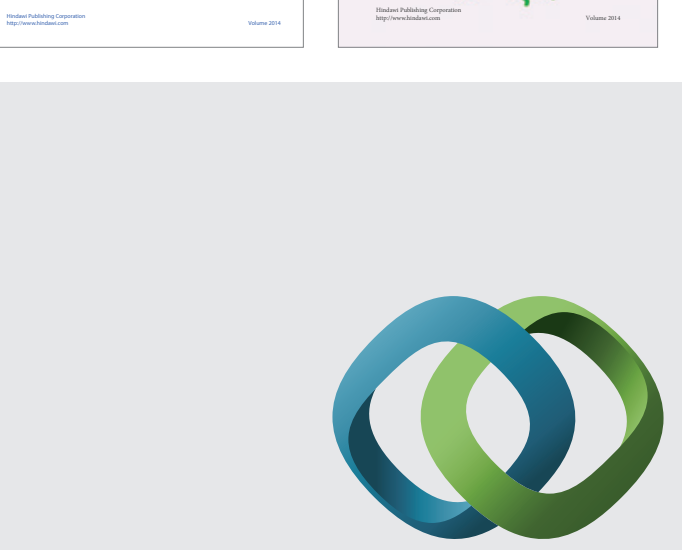

\section{Hindawi}

Submit your manuscripts at

http://www.hindawi.com
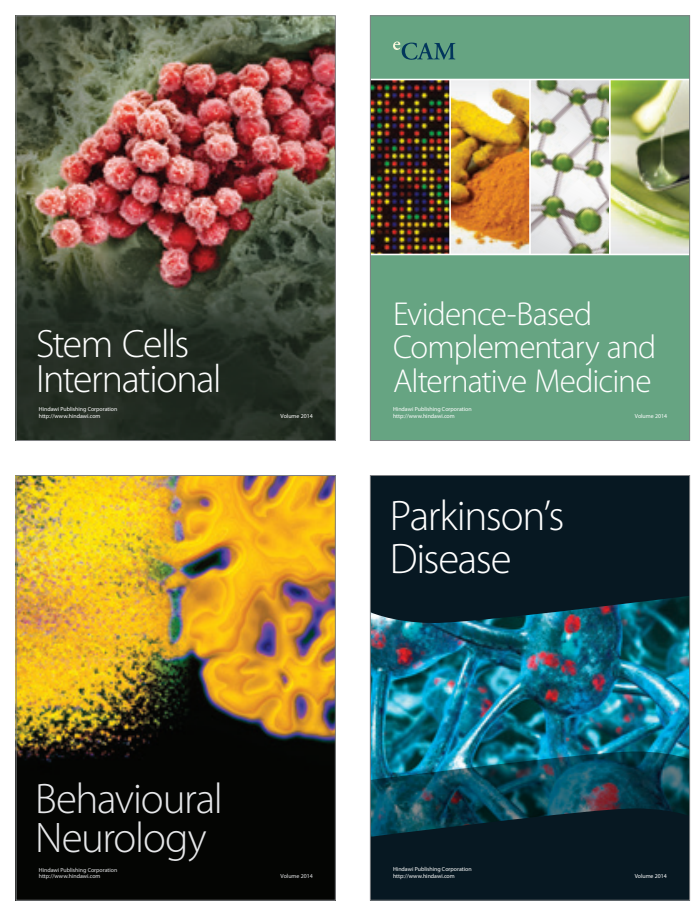

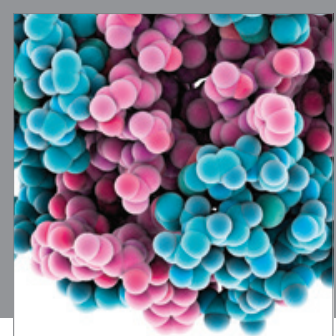

Journal of
Diabetes Research

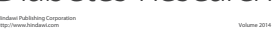

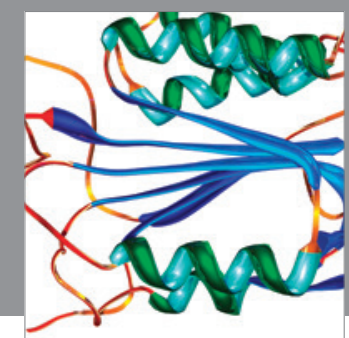

Disease Markers
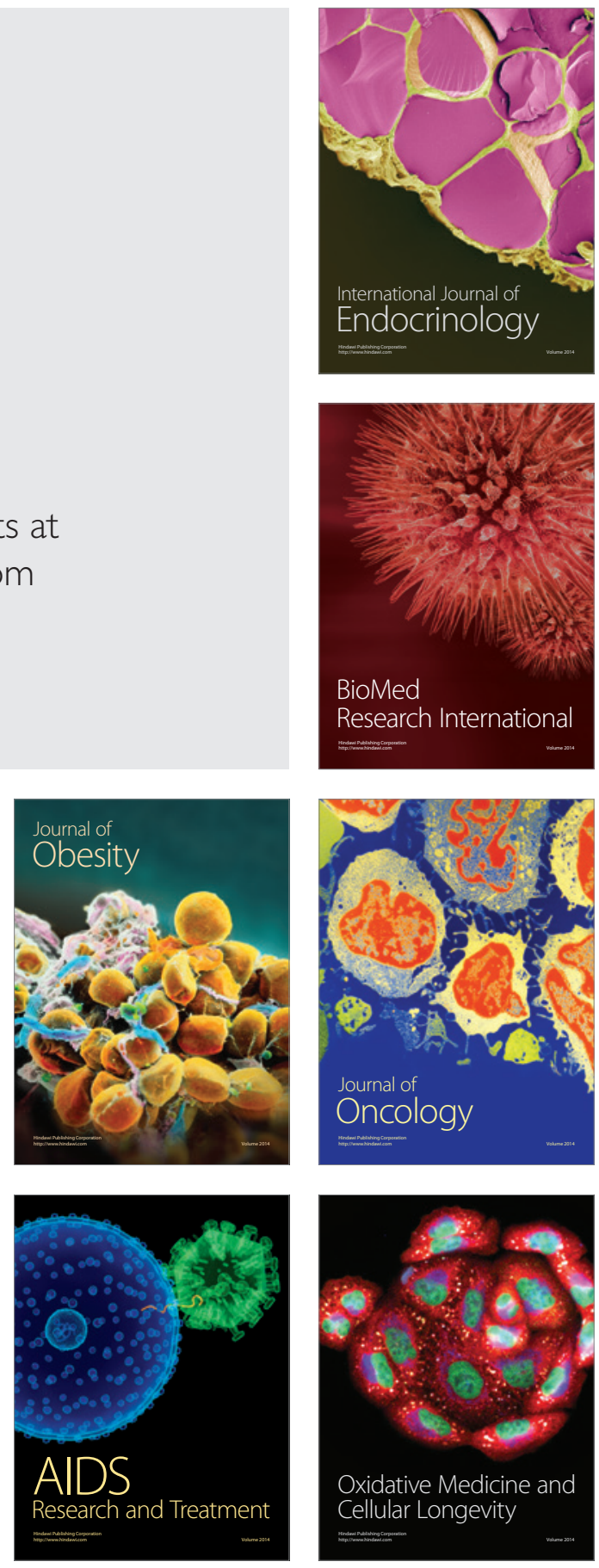